\title{
Subaperture Analysis of Polarimetric SAR Data for Iceberg Detection
}

\author{
Vahid Akbari, Anthony Paul Doulgeris, and Camilla Brekke \\ Earth Observation Laboratory \\ Department of Physics and Technology \\ University of Troms $\varnothing$-The Arctic University of Norway
}

\begin{abstract}
This paper is focused on investigations of the improved detection of icebergs in open water using spectral analysis of polarimetric SAR data. The objectives of this study are to analyze the behavior of ice objects in open water using the sublook cross-correlation magnitude (SCM). It is shown that there is an improvement of iceberg-sea contrast when the SCM is used instead of multilook intensities from the full-bandwidth data. The subband extraction in the azimuth dimension is used to test the stability of icebergs when they are observed by different azimuth view angles (i.e., looking fore or aft). Experiments are performed on full-polarimetric single-look complex (SLC) SAR data containing icebergs in open water. The results indicate that the sublook analysis has a impact on detection performance.

Index Terms-Synthetic aperture radar (SAR); polarimetry; subaperture processing; sublook cross-correlation magnitude; iceberg detection; contrast enhancement.
\end{abstract}

\section{INTRODUCTION}

As icebergs pose a significant danger for shipping and offshore oil exploration or production, any improvement in the ability to detect and track their progress along the Arctic regions would be valuable. Since spaceborne synthetic aperture radar (SAR) systems can image the ocean and their sea ice regions independent of light and cloud conditions, its potential for iceberg monitoring is a relevant application of remote sensing [1], [2].

The potential of SAR remote sensing for iceberg detection depends on the physical properties of the iceberg (such as size, shape, and structure), on the orientation relative to the radar-look direction, on the backscatter of the surrounding sea ice or open water, and also on the oceanic and meteorological conditions. Many studies have demonstrated the potential of SAR images in icebergs detection and characterization, e.g., [3], [4].

The aim of this paper is to investigate contrast enhancement based on spectral analysis of polarimetric SAR images. The motivation of using spectral analysis for detection is that point and extended targets (i.e, icebergs with different dimensions and growlers) are expected to have a spectral response that is more stable as compared to open water over the time span separating the acquisition of the subapertures, and therefore these targets will express a relatively high coherence [5]. The main objective of this study is to test the stability of icebergs from different azimuth angles. To do this, we extract the sublooks with different central frequencies and analyse the contrast measure from the sublook cross-correlation magnitude (SCM).

The paper is organised as follows: Section II presents the spectral analysis of polarimetric SAR data. Experimental results of the contrast enhancement from the SCM are given in Section III and conclusions in Section IV.

\section{Spectral Analysis of SAR Data}

This section aims at presenting two algorithms exploiting properties of the SAR image spectrum to perform iceberg detection.

The SLC data is first converted to the frequency domain by a fast Fourier transform (FFT) in azimuth dimension (or range). Then the azimuth antenna pattern (or range window) is removed from the original spectrum to get the unweighted spectrum, which is flat in the ideal case. Finally, to extract sublooks, the flat spectrum is multiplied by a rectangular weighing function to select different portions of the flat spectrum at different central frequencies. Then, the spectrum is split into sublooks, which are consequently shifted to the same center frequency to avoid linear phase terms during the cross correlation of sublooks. An inverse Fourier transform is finally applied to each sublook to obtain them in the spatial domain [6].

Let $N_{a z}$ be the number of azimuth sublooks with central frequencies $f_{c}^{(n)}$, for $n=1, \cdots, N_{a z}$, and equal bandwidths $B_{s}$.

$$
f_{c}^{(n)}=-\frac{B}{2}+\frac{B_{s}}{2}+\frac{n-1}{N-1}\left(B-B_{s}\right),
$$

where $B$ and $B_{s}$ are the full and sublook bandwidths, respectively, and $f_{c}^{(n)}$ is the central frequency of the sublook $n$. To obtain independent sublooks, $B_{s}=B / N$ [6]. The correlation among clutter samples depends on the portion of the overlapping sublook bandwidth, denoted $B_{s}$ with respect to the total available azimuth bandwidth $B$ as [6].

$$
[M]_{n, m}=\left\{\begin{array}{cc}
1-\frac{\left|f_{c}^{(m}-f_{c}^{(n)}\right|}{B_{s}}, & \text { if } \Delta f_{c}<B_{s} \\
0 & \text { otherwise }
\end{array}\right.
$$

for $n, m=1, \cdots, N$. The time separation between sublooks at central frequencies of $f_{c}^{(m)}$ and $f_{c}^{(n)}$ is given as [7]

$$
\Delta T=T_{c}^{(m)}-T_{c}^{(n)}=\frac{\lambda R\left(f_{c}^{(m)}-f_{c}^{(n)}\right)}{\left(2 V^{2}\right)}
$$


As $\Delta T$ increases, the overlapping area of subapertures decreases and open water starts to decorrelate, while a stable targets should remain coherent over the time separating the acquisition of the subapertures. We want to study the effect of splitting the bandwidth into two azimuth subapertures with respect to varying time separation between sublooks. The complex scattering vector of the extracted sublooks at different central frequencies for each pixel is given as:

$$
\underline{\mathbf{S}}=\left[\begin{array}{llll}
S_{1} & S_{2} & \cdots & S_{N}
\end{array}\right] .
$$

The cross-correlation between two sublooks at different central frequencies is calculated as [8]

$$
\rho_{n m}=<S_{n} \cdot S_{m}^{*}>\text {, }
$$

where $\rho_{n m}$ is named in [8] as internal Hermitian product (IHP), * means the complex conjugate, $S_{n}$ and $S_{m}$ are the complex scattering coefficients associated with the two generic subapertures extracted for a given pixel, $\langle\cdot\rangle$ indicates spatial sample averaging. The multilook complex (MLC) covariance matrix of the subaperture complex scattering vectors from different view (azimuth or range) angles is computed as

$$
\begin{gathered}
\mathbf{P}=\frac{1}{L} \sum_{\ell=1}^{L} \underline{\mathbf{S}}_{\ell} \cdot \underline{\mathbf{S}}_{\ell}^{H} \\
=\left(\begin{array}{cccc}
\left\langle\left|S_{1}\right|^{2}\right\rangle & \left\langle S_{1} \cdot S_{2}^{*}\right\rangle & \cdots & \left\langle S_{1} \cdot S_{N}^{*}\right\rangle \\
\left\langle S_{2} \cdot S_{1}^{*}\right\rangle & \left\langle\left|S_{2}\right|^{2}\right\rangle & \cdots & \left\langle S_{2} \cdot S_{N}^{*}\right\rangle \\
\vdots & \vdots & \ddots & \vdots \\
\left\langle S_{N} \cdot S_{1}^{*}\right\rangle & \left\langle S_{N} \cdot S_{2}^{*}\right\rangle & \cdots & \left\langle\left|S_{N}\right|^{2}\right\rangle
\end{array}\right),
\end{gathered}
$$

where $L$ is the number of pixels for spatial averaging and $(\cdot)^{H}$ denotes the Hermitian transposition operator. The diagonal elements are the multilook intensities of the sublooks and off-diagonal element are cross-correlation between sublooks with varying time separation. Each pixel in the MLC covariance matrix of the sublooks is a realization of the $N \times N$ stochastic matrix variable denoted as $\mathbf{P}$. We take the SCM as $\left|\rho_{n m}\right|=|\mathbf{P}(n, m)|=\left|\left\langle S_{n} \cdot S_{m}^{*}\right\rangle\right|$ from each element of $\mathbf{P}$ to use in determining contrast measure between iceberg and open water. For this study, the TCR is used for contrast measures as the ratio of the mean value of the target-only return to the mean value of the clutter-only return in decibel.

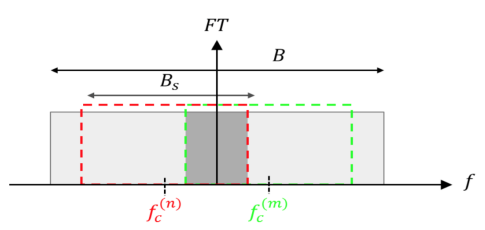

Fig. 1. Example of two generic sublooks at different central frequencies.

\section{EXPERIMENTAL RESULTS}

The processed data consist of one C-band SLC Fine QuadPol RADARSAT-2 SAR data in which visually inspected icebergs are present. That was acquired over Hopen, an island in the southeastern part of Svalbard archipelago. Fig. 2 shows the

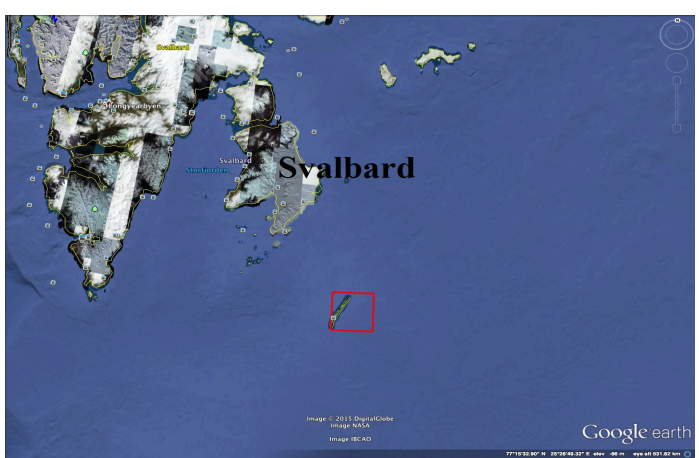

Fig. 2. Location (red box) of the RADARSAT-2 image for the area of interest, the southeast of Svalbard archipelago. Google Earth image.

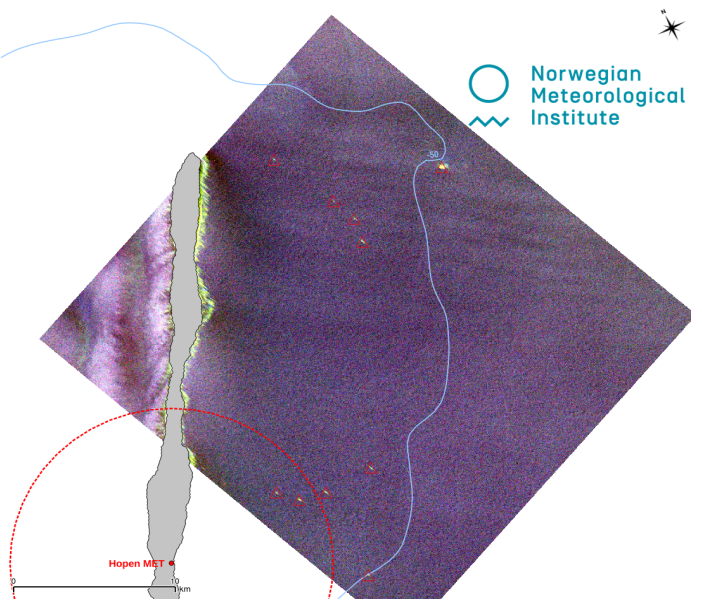

Fig. 3. Example of ten icebergs in RGB image of the quad-pol RADARSAT-2 image over Hopen from 31 July 2015.

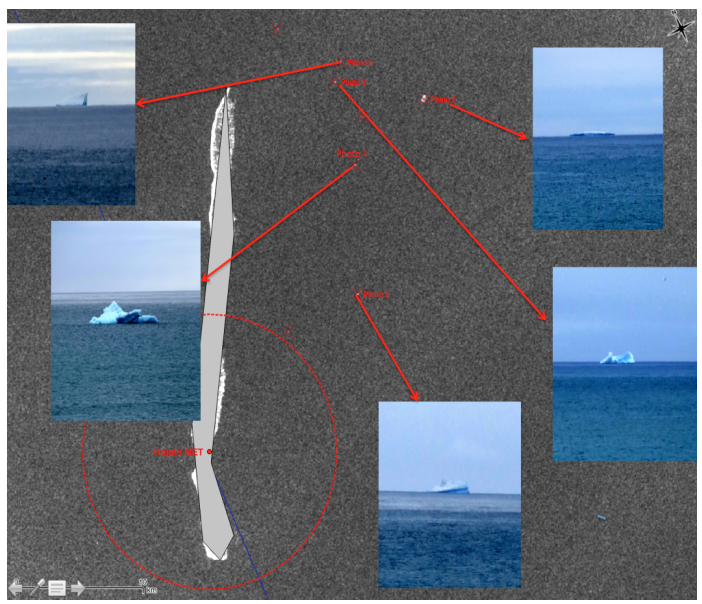

Fig. 4. Example of five icebergs in radar imagery. The figure also shows photos of the icebergs seen from the shore. Iceberg photos are courtesy of weather observing team of Norwegian meteorological institute.

locations of the RADARSAT-2 image for the area of interest on Google Earth. The Norwegian Meteorological Institute's manned weather station at Hopen has observed a number of icebergs and growlers. SAR data is characterized by a nominal 

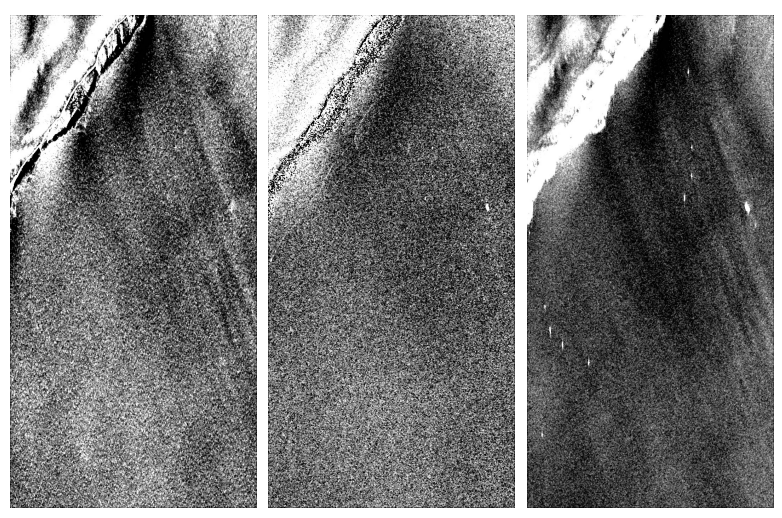

Fig. 5. Intensities corresponding to the pauli decomposition, HH+VV (left), HH-VV (middle), and HV (right) for the RGB image in Fig. 3.
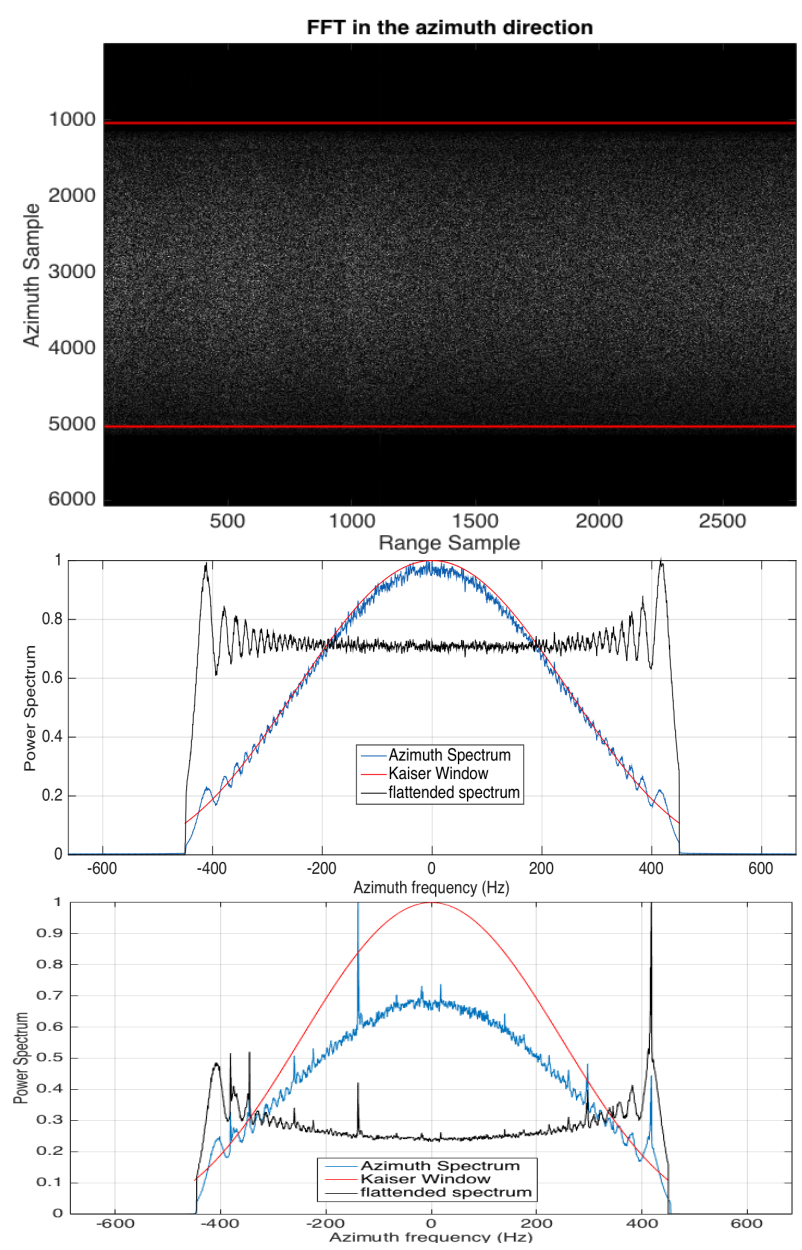

Fig. 6. Magnitude of the SLC spectrum for HH channel (top panel), Power spectrum, azimuth antenna pattern and flattened spectrum for $\mathrm{HH}$ (middle panel) and HV (bottom panel) channels. The total processed bandwidth $B$ is $900 \mathrm{~Hz}$.

spatial resolution of $4.7 \mathrm{~m} \times 5.1 \mathrm{~m}$ in slant range and azimuth, respectively. An enhanced RGB composite image (red $=\mathrm{HH}$, green $=\mathrm{HV}$, blue $=\mathrm{VV}$ ) of the PolSAR image is shown in Fig. 3. The map also shows the $50 \mathrm{~m}$ water depth contour around Hopen. In total, 10 icebergs were observed with a variety of dimensions (ranging between $30 \mathrm{~m}$ to $400 \mathrm{~m}$ in length) and typology (e.g. large, small, pinnacled, tabular, domed, etc). Fig. 4 shows the five photos of the icebergs taken across the Hopen coast.

The backscatter values over ocean clutter are expected to be higher for co-polarized channels, while the $\mathrm{VH}$ and HV signal is often hidden below the noise floor. The radar backscatter from icebergs/growlers arises dominantly from volume scattering due to the low absorption of the glacial ice which allows considerable penetration of the radar energy into the iceberg volume [3]. Fig. 5 illustrates the components of Pauli decomposition corresponding to surface scattering $(\mathrm{HH}+\mathrm{VV})$, double-bounce scattering (HH-VV), and volume scattering (HV). It is worth mentioning that depolarization can be made not only by the iceberg volume but also by the orientation of the icebergs with respect to the radar-look direction. By the dominant radar backscatter for the icebergs and weak backscattering over ocean in the HV channel, it is easy to see that the potential of the HV channel for the iceberg detection ought to be superior compared to the co-pol channels.

The spectral analysis of SAR data for iceberg detection is used to investigate the impact of the sublook extraction on detection performance. The three polarimetric channels $\mathrm{HH}$, $\mathrm{HV}$, and VH are analyzed separately. The following steps are followed to extract sublooks from the SLC data. In this paper, we confine ourselves to the subband extraction from the azimuth spectrum. The SLC data is first converted to the frequency domain by a FFT in the azimuth direction (see top panel in Fig. 6) and averaged out to obtain the azimuth power spectrum. The blue curve in Fig. 6 shows such spectrum for $\mathrm{HH}$ (middle panel) and HV (bottom panel) channels. The Kaiser weighting function has been used for a better side-lobe suppression for both range and azimuth spectral weighting as a part of the RADARSAT-2 SAR processor. An azimuth bandwidth reduction is also done with a modified Kaiser weighting function which is referred here as a the total available azimuth bandwidth $B$. The Earth's rotation causes the systematic variations of the Doppler centroid in satellite attitude as a function of time. The doppler centroid, $f_{d c}$, is shifted to zero azimuth frequency, $f_{0}$ to compensate for this effect [9]. The original spectrum is finally shifted around the peak of the Kaiser weighting function to get highest correlation coefficient between the two spectra. The azimuth antenna pattern is removed from the shifted original spectrum to obtain the unweighted and flattened spectrum (black spectra in Fig. 6). For both $\mathrm{HH}$ and $\mathrm{HV}$ channels, the ringing pattern is observed due to the signal noise at both edges of the flattened spectrum that is recommended to cut off and extract sublooks from the middle part of the specrum, at the expense of a strong resolution loss. The sublooks with a bandwidth of $B_{s}=0.5 * B$ at different central frequencies are now extracted from the flattened spectrum and are transferred back to the spatial domain by the inverse Fourier transform.

The sublook cross-correlations are computed for each pair of sublooks with different time separations, defined as $\Delta T$ 

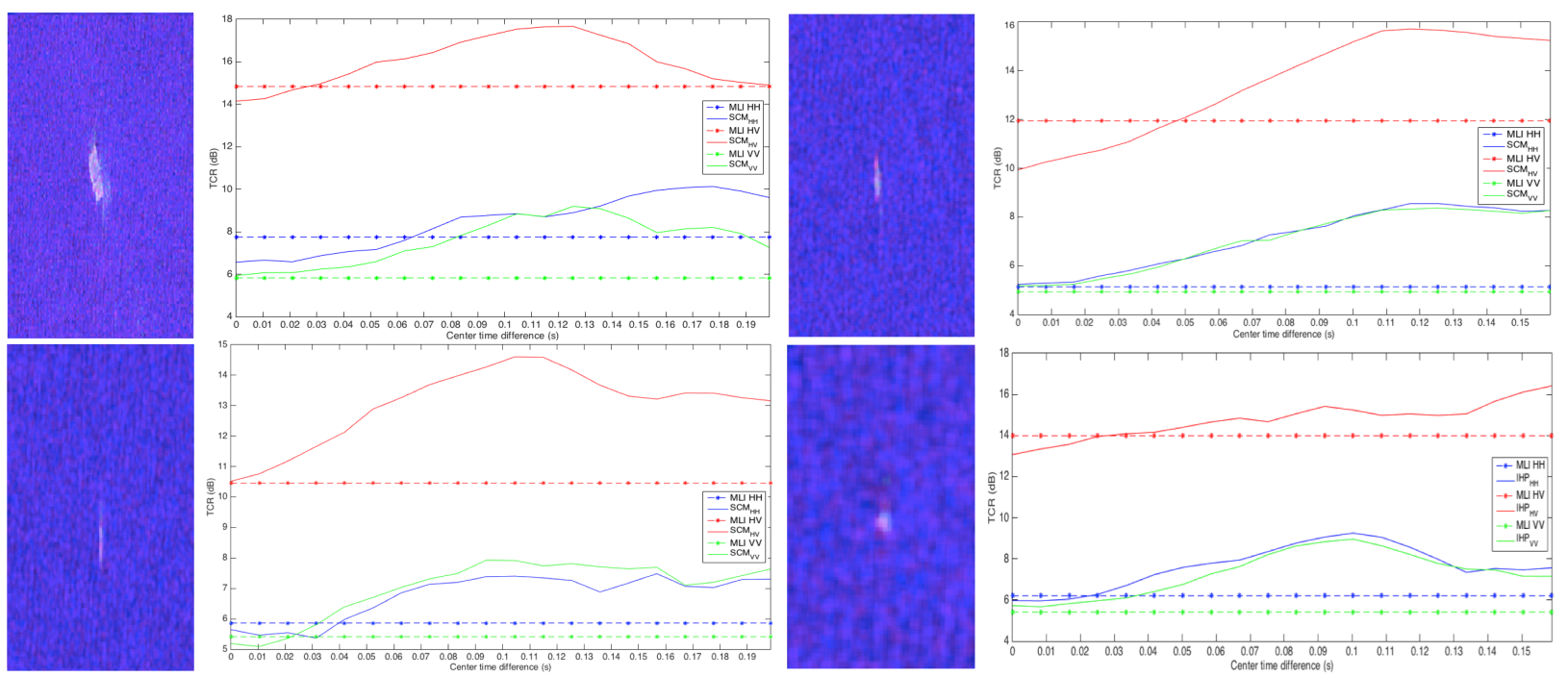

Fig. 7. TCR versus time separation between sublooks, $\Delta T$. SCM with a subaperture bandwidth of $B_{s}=0.5 * B$ compared to MLI for the four icebergs indicated in Fig. 3 in three polarimetric channels.

in (3). Hence, it ranges from 0 to 0.19 seconds, where the subapertures overlap goes from $100 \%$ to $0 \%$. We finally compare multilook intensity (MLI) and SCM with respect to measures of the contrast between the icebergs and the surrounding background. In particular, we investigate the contrast measures by the TCR as a function of time separations between sublooks. Fig. 7 illustrates the contrast enhancement between the icebergs and the surrounding open water as a function of $\Delta T$ for the four icebergs in Fig. 3. For each polarization channel, the largest peak indicates maximum contrast enhancement and accordingly the optimal azimuth subaperture separation. The results show that both the optimal subaperture separation and the maximum contrast enhancement varies between the polarimetric channels. HV polarization has the highest TCR in all cases. For $\Delta T=0$, a reduced TCR for all polarimetric channels is achieved for SCM compared to MLI, as expected. As $\Delta T$ increases, TCR increases in most cases. As seen in Fig. 7 (top left), from some point in HV channel, the TCR starts to decrease again. This is likely due to multiple reflections from two different azimuth angles or different orientation angle relative to the radar look view. The interpretation of the TCR results gets complicated if icebergs drift throughout the illumination time. If the dominant scattering mechanism is a single-bounce or double-bounce scattering, the phase difference of the sublooks may have a deterministic form, and the sublooks are correlated. However, in practice, multiple reflections from various parts of the icebergs, specially large ones, contribute to the radar backscatter, so that even for a small difference in azimuth look angle, the phases of received signal tend to be random and uncorrelated between subapertures.

\section{CONCLUSION}

This paper showed the improved detection of icebergs in open water using spectral analysis of polarimetric SAR data. The stability of icebergs was tested by cross-correlating sublooks with different azimuth angles. It was shown that the enhancement of iceberg-sea contrast is obtained using the sublook cross-correlation magnitude.

\section{ACKNOWLEDGEMENTS}

The authors wish to thank Thomas Kræmer at the Arctic University of Norway for his valuable discussions and technical helps. RADARSAT-2 Data and Products (CMacDonald, Dettwiler and Associates Ltd. (2015)-All Rights Reserved. RADARSAT is an official mark of the Canadian Space Agency.

\section{REFERENCES}

[1] M. Denbina, "Iceberg detection using compact polarimetric synthetic aperture radar," Ph.D. dissertation, University of Calgary, August 2014.

[2] W. Dierking and C. Wesche, "C-band radar polarimetry: Useful for detection of icebergs in sea ice?" IEEE Trans. Geosci. Remote Sens., vol. 52, no. 1, pp. 25-37, Jan 2014.

[3] D. Power, J. Youden, K. Lane, C. Randell, and D. Flett, "Iceberg detection capabilities of radarsat synthetic aperture radar," Canadian Journal of Remote Sensing, vol. 27, no. 5, pp. 476-486, 2001.

[4] R. Gill, "Operational detection of sea ice edges and icebergs using sar," Canadian journal of remote sensing, vol. 27, no. 5, pp. 411-432, 2001.

[5] A. Marino, M. J. Sanjuan-Ferrer, I. Hajnsek, and K. Ouchi, "Ship detection with spectral analysis of synthetic aperture radar: A comparison of new and well-known algorithms," Remote Sensing, vol. 7, no. 5, pp. 5416-5439, 2015.

[6] M. J. Sanjuan-Ferrer, I. Hajnsek, K. P. Papathanassiou, and A. Moreira, "A new detection algorithm for coherent scatterers in SAR data," IEEE Trans. Geosci. Remote Sens., vol. 53, no. 11, pp. 6293-6307, Nov 2015.

[7] K. Ouchi and H. Wang, "Interlook cross-correlation function of speckle in SAR images of sea surface processed with partially overlapped subapertures," IEEE Trans. Geosci. Remote Sens., vol. 43, no. 4, pp. 695-701, 2005.

[8] J. Souyris, C. Henry, and F. Adragna, "On the use of complex SAR image spectral analysis for target detection: assessment of polarimetry," IEEE Trans. Geosci. Remote Sens., vol. 41, no. 12, pp. 2725-2734, 2003.

[9] C. Brekke, S. N. Anfinsen, and Y. Larsen, "Subband extraction strategies in ship detection with the subaperture cross-correlation magnitude," IEEE Geosci. Remote Sensing Lett., vol. 10, no. 4, pp. 786-790, 2013. 